\title{
Energy-based seismic collapse criterion for ductile planar structural frames
}

Derya Deniz ${ }^{\mathrm{a}, *}$, Junho Song ${ }^{\mathrm{b}}$, and Jerome F. Hajjar ${ }^{\mathrm{c}}$

\begin{abstract}
One of the most common approaches to assess the collapse capacity of structures under earthquakes is incremental dynamic analysis (IDA), which tracks relationship between a structural damage measure and a ground motion intensity measure by so-called IDA curves. This IDA approach often uses collapse criteria given in terms of a large value of the maximum inter-story drift ratio, plastic hinge formations at structural components, or flattening of the IDA curve. However, these collapse criteria may not accurately represent the overall collapse behavior of structural systems due to redistribution and variation of damage within the structure. Moreover, collapse predictions by these subjective collapse limit-states are found to be sensitive to the assumed threshold values and to the characteristics of IDA curves. For more accurate assessment of collapse capacity and the likelihood of the collapse, this paper proposes a new collapse criterion that describes dynamic instability of frame structures in terms of the balance between the energies from the applied gravity loads and input ground motions. The collapse criterion is developed for planar frames under horizontally applied earthquakes and then tested using computational models of collapse behavior of ductile steel frame structures, which are validated by experiments reported in the literature. The collapse prediction results by the developed collapse criterion and existing criteria are compared in order to investigate sensitivity of the prediction results with respect to threshold values used by existing approaches. The results show that the proposed energy-based seismic collapse criterion is a more reliable option for assessing structural collapse of planar frames. The energy-based criterion can represent global dynamic instability of structural system more effectively by using aggregated quantities of energy responses of structural components instead of using assumed threshold values for structural responses such as story drifts.
\end{abstract}

KEYWORDS: incremental dynamic analysis, collapse criterion, collapse limit-state, collapse modeling, seismic energy, gravity energy

\section{Introduction}

Modern seismic design provisions help enhance life safety of building occupants during a strong earthquake-shaking event by ensuring acceptably small probability of structural collapse. Accurate estimation of collapse likelihood of buildings under seismic excitations has recently become critical in efforts to promote hazard-resilience of the society, especially in developing national building codes, regional emergency response plans, and risk management strategies. Despite extensive research conducted in recent years, current collapse limit-states adopted in 
literature do not necessarily represent actual collapse at global scale but instead often focus on local damage at so-called near collapse or collapse prevention states.

Global collapse capacity of a frame structure under seismic excitation can be defined as the structural resistance just before the structure shows dynamic instability, that is, the structure, or any significant part of it, is not able to find a new equilibrium configuration, therefore loses the ability to sustain the gravity loads. A building structure is considered dynamically unstable when the structural system starts to show boundless story drifts. One of the most common approaches to determine the global collapse capacity of a structural system under earthquake excitations is incremental dynamic analysis (IDA) [1,2]. This approach constructs so-called "IDA curves" to identify relationship between an intensity measure (IM) (e.g., spectral acceleration of an earthquake input) and a damage measure (DM) or engineering decision parameter (EDP) (e.g., maximum inter-story drift ratio) through nonlinear dynamic analyses under ground motions at incrementally increased intensity levels. Since a dynamically unstable system exhibits a large increase in the structural response for a small increase in the ground motion intensity, the IDA curve becomes flattened, which indicates the collapse of the structural system. However, the flattening of IDA curves may not be clear or curves may show unusual behavior, such as nonmonotonic behavior and discontinuities. To identify the collapse state despite such challenges, some collapse criteria have been proposed by Vamvatsikos and Cornell [1]: The building's global drift capacity against collapse was defined as the maximum story drift ratio at which the slope of the IDA curve reduces to $20 \%$ of the initial slope ("IM-based" criterion), but if IDA curve does not fulfill the IM-based criterion, then one checks if the drift ratio exceeds an assumed global drift capacity, e.g., 10\% ("DM-based" criterion).

Although this approach is one of the state-of-art procedures in structural collapse capacity assessment, it is noted that this procedure may have the following limitations: First, the IDA curve could flatten due to large residual DMs, which may not indicate the inability to sustain gravity loads necessarily. In addition, the IM-based and DM-based criteria are subjective limitstates relying on some assumed threshold values instead of the actual occurrence of dynamic instability. Therefore, the collapse capacity (both in terms of IM and DM) identified by these criteria could be sensitive to the assumed threshold values. In other terms, depending on the assumed value, these criteria could provide different evaluations of collapse capacity, which may not be the actual capacity against dynamic instability necessarily. Moreover, IDA curves may show characteristics in which the collapse criteria is breached, but then the structure remains stable at higher levels of loading. In that case, the aforementioned criteria may provide more than one collapse capacity for the applied ground motion that makes collapse capacity assessment elusive.

It is noted that existing approaches often use maximum (peak) response of the structure as the damage measure such as maximum inter-story drift. Since such peak responses can vary in the structure, one may obtain different estimation on damage states, e.g., light, moderate, severe damage and collapse, depending on the selected location at which the peak response is measured. To address this issue, it is desirable to evaluate cumulative response measure for the global system instead of measuring local peak responses only. Since the cumulative measures are loadpath dependent, they are expected to help consider the damage history and pattern due to cyclic seismic loading. Accumulated plastic deformation and the hysteretic energy are commonly used for calculating cumulative damage indices [3-6]. However, most of these cumulative measures are usually assessed only at local level, and thus they are most appropriate for evaluating losses in resistance of individual elements prior to collapse rather than the global system. 
This paper presents an energy-based collapse analysis of structures at the system level to identify global dynamic instability of building structures under variable seismic excitations. and variation of each individual component-damage within the structural system, they provide global information to represent cumulative structural damage due to cyclic loading up to and including collapse. In this work, nonlinear dynamic analyses are first performed for three experimental case studies for steel frames reported in the literature using OpenSees, an objectoriented software framework developed by Pacific Earthquake Engineering Center (PEER) [7]. Using the OpenSees computational models validated by these corresponding experimental results, a parametric investigation is conducted to develop a new energy-based collapse limit-state for planar frames under horizontally applied seismic loadings to identify dynamic instability due to loss of structural resistance against the gravity loads. The selected case studies are then used to test the new collapse limit-state. Collapse evaluations by the new collapse criterion are then compared to those resulting from typical use of IDAs employing either DM-based or IM-based criteria. In particular, sensitivity analyses are performed on the assumed threshold values used in the conventional criteria to clearly demonstrate the merits of the proposed energy-based collapse criterion.

\section{Validated computational simulations of collapse}

In order to develop a new collapse limit-state for more reliable structural collapse assessment under cyclic dynamic loadings, it is necessary to build validated computational models that simulate structural collapse accurately. This is because it is impossible to obtain enough amount of real or experimental data required to develop such a collapse limit-state. This study selected three sets of steel frame experimental shake-table tests by Kanvinde [8], Rodgers and Mahin [9], and Lignos et al. [10] to develop validated computational models with 1-story, 2-story, and 4story structures, respectively, representing three examples of ductile steel frames with different geometries, load settings, hysteretic behaviors (see Table 1). While the ductile-baseline (DB) case study by Rodgers and Mahin [9] has a first-mode dominant period of 0.65 seconds, the case studies by Kanvinde [8] and Lignos et al. [10] have a similar first-mode dominant period between 0.40 and 0.50 seconds, but all cases have different hysteretic behaviors resulting in different strength ratios and strain magnitudes. OpenSees models comparable to the models developed in the original experimental studies were developed for each test case study, and were then validated against the corresponding experimental tests results. A sidesway collapse mechanism (i.e., significant growth of lateral story drifts under seismic forces) was the dominant failure mode in the collapse experiments.

Table 1. Experiment details for three steel frame collapse shake-table tests [1 in $\approx 2.54 \mathrm{~cm}$ ].

\begin{tabular}{|c|c|c|c|c|c|c|c|c|c|}
\hline Experiment & $\begin{array}{l}\frac{0}{\tilde{J}} \\
\tilde{n}\end{array}$ & 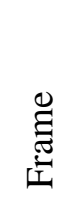 & 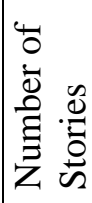 & 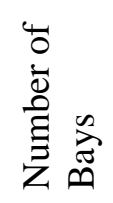 & 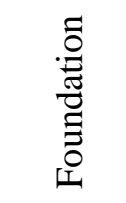 & 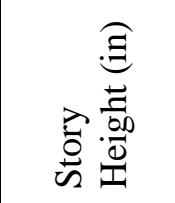 & 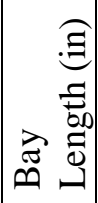 & 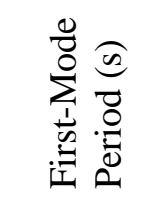 & 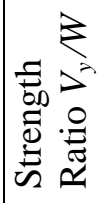 \\
\hline Kanvinde [8] & $\sim 1 / 13$ & $3 \mathrm{D}$ & 1 & 1 & Fixed & 12 & 24 & $0.48 \sim 0.50$ & 0.6 \\
\hline Rodgers \& Mahin (DB case)[9] & $1 / 3$ & $3 \mathrm{D}$ & 2 & 1 by 2 & Pinned & 108 & 54 & \begin{tabular}{|l|}
0.65 \\
\end{tabular} & 0.5 \\
\hline Lignos et al. [10] & $1 / 8$ & $2 \mathrm{D}$ & 4 & 2 & Fixed & $20\left(1^{\text {st }}\right), 18$ & 45 & $0.40 \sim 0.46$ & 0.2 \\
\hline
\end{tabular}




\subsection{Basic assumptions for collapse simulations}

Prior studies on collapse assessment of steel structures have often used macro-models incorporating softening responses to simulate the effects of significant yielding and fracture or cyclic deterioration, coupled with geometric nonlinear behavior [8-12]. This work uses macromodel formulations that reflect the models used by the experimentalists for consistency and successful validation. This study therefore focuses on developing two-dimensional nonlinear dynamic finite element models of the selected steel moment-resisting frames using elastic beam elements for the girders and columns, coupled with the use of uniaxial, zero-length momentrotation relations at the element ends.

\subsection{Collapse case studies}

Of the three selected experiments [8-10] used to validate the computational models for establishing collapse criteria [13,14], two of the test cases are described here in detail: a onestory steel frame test by Kanvinde [8] and a four-story steel frame test by Lignos et al. [10].

Kanvinde [8] conducted shake-table tests on a single-story steel specimen configuration measuring 12" by $24 "$ [ 30.48 cm by $60.96 \mathrm{~cm}]$ in plan (the longer dimension aligned in the direction of motion) and 10" [ 25.40 cm] in clear height to investigate dynamic instability of structures caused by earthquake excitations. The specimen configuration was in the form of four flat steel columns connected to a base plate. A steel mass on top served as a rigid diaphragm as shown in Fig. 1a. The columns have a cross-section of 1/8" (along the direction of motion) by $1 "$ [ 0.32 cm by $2.54 \mathrm{~cm}]$ with $1 / 2 "[\sim 1.27 \mathrm{~cm}]$ holes drilled at the column ends to introduce locations of concentrated plasticity. The design was chosen to provide a simple validation platform to exercise the geometric and material nonlinear components of the collapse formulation by comparing to a testbed with clean, unidirectional behavior. The structure failed in a story mechanism, with columns bending in double-curvature, with plastic-hinges at the top as well as the bottom of the columns. A structural model in OpenSees was built (Fig. 1b) following the two-dimensional computational model details provided by Kanvinde [8]. Elastic elements were assigned to the columns and beam, and the beam was modeled as a rigid link with a large moment of inertia and sectional area. Concentrated bi-directional nodal masses (each $0.2072 \mathrm{lbs}-\mathrm{s}^{2} / \mathrm{in}\left[\sim 3.7002 \mathrm{~kg}-\mathrm{s}^{2} / \mathrm{m}\right]$ in both the horizontal and vertical directions) were placed at the two ends of the top beam. Rayleigh damping of $2 \%$ was applied on both the mass and the tangent stiffness (i.e., the stiffness at each iteration of one time step of analysis) of the frame model. Mass and stiffness damping coefficients were obtained based on the first and second modes. Inelastic SDOF zero-length rotational springs were modeled at the plastic locations at the ends of the columns by assuming a Giufré-Menegotto-Pinto plasticity model [15]. For this purpose, the corresponding material model "Stee102" in OpenSees (see Fig. 1c) was utilized for the spring hysteretic response. The following values for the parameters used in the spring model were selected to match available test experiment results: an initial stiffness $\left(K_{i}\right)$ of $8.0 \mathrm{kips}-\mathrm{in} / \mathrm{rad}$ [ 0.9039 kN-m/rad ], a yield moment $\left(M_{y}\right)$ of 0.2398 kips-in $[\sim 0.0271 \mathrm{kN}-\mathrm{m}]$, a softening amount $(\alpha)$ of $0.75 \%$ after yielding, and curvature values of $16.8\left(R_{0}\right), 0.925\left(R_{1}\right)$, and $0.15\left(R_{2}\right)$ that determine the transition from elastic to plastic branches. A co-rotational formulation was used to account for nonlinear geometric effects [16]. The modal analysis provided a first mode period that matched closely with the test frame, which is $0.443 \mathrm{sec}$. As one of a series of comparisons made with the results presented by Kanvinde [8], nonlinear dynamic analysis was used with an input ground motion from the 1994 Northridge Earthquake record at Obregon Park; this constituted test case No. 11 in Fig. 1d. The Newmark- $\beta$ average acceleration method was 
used to compute the time integration. The model used in this work matched closely with the available experiment and computational results presented by Kanvinde [8] up to and including near-collapse.

Lignos et al. [10] performed a series of collapse shake-table tests of a 4-story, 2-bay steel frame with reduced-beam sections (RBS) at 1/8 scale. Fig. 2a [17] shows the setup of the test frame on the NEES mass simulator at the University at Buffalo. The steel specimen consists of elastic members with plastic hinges at the ends. The mass simulator is connected to the test frame by means of axially rigid horizontal links through which the simulator transfers P-Delta effects acting as leaning columns on the test frame. An analytical clear-span model for the 1/8 scale 4-story test frame was developed in OpenSees (see Fig. 2b) considering the distance between inside surfaces of the span supports: 19.75 in $[\sim 50.17 \mathrm{~cm}]$ for $1^{\text {st }}$ story clear height, 15.75 in $[\sim 40.00 \mathrm{~cm}]$ for height of other stories, and 42.75 in $[\sim 108.58 \mathrm{~cm}]$ for the clear-bay length in the frame. Similar to the model built for the case study by Kanvinde [8], concentrated nodal masses were placed bi-directionally at each floor of the leaning columns: 8.8 kips [ 39.14 $\mathrm{kN}]$ at roof and $8.6 \mathrm{kips}[\sim 38.25 \mathrm{kN}]$ at the $1^{\text {st }}, 2^{\text {nd }}$ and $3^{\text {rd }}$ floors. Rayleigh damping of $1.5 \%$ was applied both on the mass and on the tangent stiffness of the frame model. Since unexpected friction damping was observed at the spherical hinges of the mass simulator gravity links during the experiment, a friction rotational element at the ends of each gravity link was also considered in the analytical model, following the study by Lignos and Krawinkler [18]. Rotational springs were used to model the plastic hinges in the frame with a modified Ibarra-Krawinkler deterioration model available as "Bilin" in OpenSees [10,18,19] (see Fig. 2c), calibrated based on a steel component database of steel beams with RBS connections subjected to cyclic loading. The parameters proposed by Lignos et al. [10,18] in Table 2 were used for modeling rotational springs, where $M_{y}$ is the yield moment strength; $K_{i}$ is the initial stiffness; $M_{c} M_{y}$ is the postyielding moment ratio; $\lambda$ is the cyclic deterioration parameter lambda; $\theta_{p}$ is the plastic rotational capacity; $\theta_{p c}$ is the post-capping rotational capacity; $M_{r e s}$ is the residual strength, $\theta_{u}$ is the ultimate rotation; $D$ is the rate of cyclic component deterioration; and $c$ is the rate of component deterioration. Furthermore, offsets from the connections were applied to take the RBS location into account following the method used by Lignos et al. [10,18]. Nonlinear geometric effects were considered using a corotational formulation [16]. The modal analysis provided a first-mode period close to the period observed during the experiment, which is 0.438 seconds. Adopting the same numerical procedure used in the test case study by Kanvinde [8], a series of dynamic analyses were performed sequentially using the OpenSees model and running the Canoga Park record from the 1994 Northridge Earthquake at the scale factors of 0.4, 1.0, 1.5, 1.9, and 2.2 following the experiment procedure. The OpenSees results were compared with those of the experimental for the test frame No. 1 and computational results of Lignos et al. [10,18], and showed a good match especially in terms of the intensity scale factor causing the dynamic instability (see Fig. 3). 
Table 2. Model parameter values used for the rotational springs in the mathematical model developed for the shake-table test by Lignos et al. [10,18] [1 kips-in $\approx 0.1130 \mathrm{kN}-\mathrm{m}]$.

\begin{tabular}{|l|c|c|c|c|c|c|c|c|c|c|}
\hline Location & $\begin{array}{c}M_{y} \\
\text { (kips-in) }\end{array}$ & $\begin{array}{c}K_{i} \\
\text { (kips-in/rad) }\end{array}$ & $M_{c} / M_{y}$ & $\lambda$ & $\begin{array}{c}\theta_{p} \\
(\mathrm{rad})\end{array}$ & $\begin{array}{c}\theta_{p c} \\
(\mathrm{rad})\end{array}$ & $\begin{array}{c}M_{\text {res }} \\
(\mathrm{kips}-\mathrm{in})\end{array}$ & $\begin{array}{c}\theta_{u} \\
(\mathrm{rad})\end{array}$ & $D$ & $c$ \\
\hline C1B & 34.5 & 25700 & 1.10 & 26.0 & 0.050 & 2.0 & 0 & 0.4 & 1 & 1 \\
\hline C1T,C2, C3B & 27.0 & 20631 & 1.10 & 26.0 & 0.050 & 2.0 & 0 & 0.4 & 1 & 1 \\
\hline C3T, C4 & 14.2 & 11200 & 1.08 & 18.2 & 0.055 & 2.4 & 0 & 0.4 & 1 & 1 \\
\hline B1, B2 & 19.8 & 13000 & 1.10 & 36.0 & 0.050 & 1.6 & 0 & 0.4 & 1 & 1 \\
\hline B3, B4 & 14.2 & 11200 & 1.08 & 18.2 & 0.055 & 2.4 & 0 & 0.4 & 1 & 1 \\
\hline
\end{tabular}

\subsection{Virtual simulations of collapse}

In order to identify new collapse limit-states and important parameters in the collapse assessment of structures subjected to seismic loads, extensive IDAs were performed using the validated OpenSees simulation models, i.e. a large sample of multiple DMs and corresponding IMs are obtained for multiple far-field ground motions provided by Haselton and Deierlein [20]. A set of 39 far-field ground motion pairs (two-lateral components) recorded at sites located within $10 \mathrm{~km}$ of fault rupture (totally $39 \times 2=78$ ground motion records) were used in the study. Records were selected from strong earthquake ground motions with a moment magnitude changing from 6.5 to 7.9 [20].

The new criterion has been tested and validated on the validated 2D- computational models of collapse experiments using the 78 ground motion records by Haselton and Deierlein [20]. Moreover, comprehensive parametric studies were performed on the test case study of Lignos et al. [10] under the same 78 far-field ground motion set [20] to study sensitivity of collapse predictions to the system characteristic such as story height, bay length, mass and damping, and component properties such as yield moment strength, initial stiffness, ratio of capping moment to yield moment, plastic rotational capacity, post-capping rotational capacity [13]. Results show that the new collapse criterion is able to predict collapse for the 26 different analytical models developed for the case study with a first-mode dominant period changing from 0.20 to 0.70 seconds [13]. The new criterion therefore should be able to address at least ductile planar multistory moment frame structures subjected to unidirectional horizontal earthquake loads with comparable hysteretic behavior to the collapse experiments considered in this study.

\section{Energy-based collapse criterion}

It is well established in the literature that inertia and damping forces can stabilize a structure even if the structure has indications of static instability during dynamic motion [21]. Thus, interstory drift or the rank of the tangent stiffness matrix are not complete indicators of incipient collapse. In this work, based in part on parametric investigations of the validated computational models presented above, a new dynamic-instability-based collapse criterion is developed in terms of energies from the input ground motions and the gravity loads. The equation of motion at time $t$ for a multi-degree-of-freedom (MDOF) structure under horizontal earthquake loads and gravity loads is:

$$
\underline{\underline{M}} \underline{\ddot{u}}(t)+\underline{\underline{C}} \underline{\dot{u}}(t)+\underline{f_{S}}(t)=-\underline{\underline{M}} \underline{\underline{T}} \underline{\ddot{u}}_{F}(\mathrm{t})
$$

where $\underline{u}$ is the relative nodal displacement vector, $\underline{\dot{u}}$ is the relative nodal velocity vector, $\underline{\ddot{u}}$ is 
the relative nodal acceleration vector, $\underline{\underline{M}}$ is the structural mass matrix, $\underline{\underline{C}}$ is the structural damping matrix, $\underline{f}_{S}$ is the structural restoring force vector, $\underline{\ddot{u}}_{F}$ is the acceleration vector of the horizontal ground motion and gravity, and $\underline{\underline{T}}$ is the transformation matrix mapping the input accelerations to the appropriate mass quantities in the relevant directions for each acceleration quantity.

A useful insight into the dynamic instability of structures can be gained by investigating energy balance of a structural system under dynamic and gravity forces. If one takes the integral of each term in Eq. (1) with respect to $\underline{u}$, the energy balance of the structural system can be derived as [22]:

$\int_{\underline{u}(0)}^{\underline{u}(t)}(\underline{\underline{M}} \underline{\ddot{u}}(t)) \cdot d \underline{u}+\int_{\underline{u}(0)}^{\underline{u}(t)}(\underline{\underline{C}} \underline{\dot{u}}(t)) \cdot d \underline{u}+\int_{\underline{u}(0)}^{\underline{u}(t)} \underline{f}_{S}(t) \cdot d \underline{u}=-\int_{\underline{u}(0)}^{\underline{u}(t)}\left(\underline{\underline{M}} \underline{T} \underline{\ddot{u}_{F}}(t)\right) \cdot d \underline{u}$

Describing the integrals as corresponding energy components of a structural system, Eq. (2) becomes:

$$
E_{K}+E_{D}+E_{S}=E_{I}
$$

where $E_{K}$ is the relative kinetic energy, $E_{D}$ is the damping energy, $E_{S}$ is the strain energy, and $E_{I}$ is the relative dynamic input energy. Using $d \underline{u}=\underline{\dot{u}}(t) d t$ and $d \underline{\dot{u}}=\underline{\ddot{u}}(t) d t$ the energy components in Eq. (3) can be derived as follows:

$$
\begin{gathered}
E_{K}=\int_{\underline{\underline{u}}(0)}^{\underline{u}(t)}(\underline{\underline{M}} \underline{\ddot{u}}(t)) \cdot d \underline{u}=\int_{0}^{t} \underline{\dot{u}}(t)^{T} \underline{\underline{M}} \underline{\ddot{u}}(t) d t=\int_{\underline{\dot{u}}(0)}^{\underline{\dot{u}}(t)} \underline{\dot{u}}(t)^{T} \underline{\underline{M}} d \underline{\dot{u}}=\frac{1}{2} \underline{\dot{u}}(t)^{T} \underline{\underline{M}} \underline{\dot{u}}(t) \\
E_{D}=\int_{\underline{u}(0)}^{\underline{u}(t)}(\underline{C} \underline{\dot{u}}(t)) \cdot d \underline{u}=\int_{0}^{t} \underline{\dot{u}}(t)^{T} \underline{\underline{C}}(t) \underline{\dot{u}}(t) d t \\
E_{S}=\int_{\underline{u}(0)}^{\underline{u}(t)} \underline{f_{S}}(t) \cdot d \underline{u}=\int_{0}^{t} \underline{\dot{u}}(t)^{T} \underline{f_{S}}(t) d t \\
E_{I}=-\int_{\underline{u}(0)}^{\underline{u}(t)}\left(\underline{\underline{M}} \underline{\underline{T}} \underline{\ddot{u}}_{F}(t)\right) \cdot d \underline{u}=-\int_{0}^{t} \underline{\dot{u}}(t)^{T} \underline{\underline{M}} \underline{\underline{T}} \underline{\ddot{u}}_{F}(t) d t
\end{gathered}
$$

If Rayleigh damping is adopted, the damping matrix $\underline{C}$ can be decomposed as:

$$
\underline{\underline{C}}=a_{o} \underline{\underline{M}}+a_{1} \underline{\underline{K}}(t)
$$

where $a_{o}$ and $a_{1}$ are coefficients determined from the first two dominant frequencies of the structural system, and $\underline{\underline{K}}$ can be initial elastic stiffness $\underline{\underline{K_{e}}}$ or tangent stiffness $\underline{\underline{K_{t}}}$. This study computes the tangent stiffness at each time step to construct the Rayleigh damping matrix for a more realistic description of the near-collapse behavior of the structure. Using the model in Eq. (8), the damping energy $E_{D}$ can be derived in terms of the mass and stiffness matrices:

$$
\begin{aligned}
E_{D} & =\int_{\underline{u}(0)}^{\underline{u}(t)}\left(\left[a_{o} \underline{\underline{M}}+a_{1} \underline{\underline{K_{t}}}(t)\right] \underline{\dot{u}}(t)\right) \cdot d \underline{u} \\
& =\int_{0}^{t} \underline{\dot{u}}(t)^{T} a_{o} \underline{\underline{M}} \underline{\dot{u}}(t) d t+\int_{0}^{t} \underline{\dot{u}}(t)^{T} a_{1} \underline{\underline{K_{t}}}(t) \underline{\dot{u}}(t) d t
\end{aligned}
$$

The strain energy $E_{S}$ is the total energy dissipation under the force-deformation curve of each component considering the yield displacement and the loading and displacement directions of 
each component within the system. It can be divided into two parts: elastic strain energy $E_{E}$, and hysteretic energy $E_{H}$ (plastic strain energy), i.e., $E_{S}=E_{E}+E_{H}$ [22]. Note that $E_{S}=E_{E}$ for members showing elastic behavior.

While the accelerations for the horizontal earthquake excitation $\ddot{u}_{E Q}$ vary over time, the gravity loads applied on the structure remains constant, i.e.,

$$
\underline{\ddot{u}}_{F}=\left[\begin{array}{c}
\ddot{u}_{E Q}(t) \\
g
\end{array}\right]
$$

where, $g$ is acceleration of gravity $\left(-386.2 \mathrm{in} / \mathrm{sec}^{2}\right)$. Consequently, the input energy in Eq. (7), $E_{I}$ can be separated into the dynamic input energy due to seismic actions, $E_{E Q}$, and gravity energy due to the applied gravity loads on the structure, $E_{G}$, that is,

$$
\begin{gathered}
E_{E Q}=-\int_{\underline{u}(0)}^{\underline{u}(t)} \underline{\underline{M}} \underline{T} \ddot{u}_{E Q}(t) d \underline{u}=-\int_{0}^{t} \underline{\dot{u}}(t)^{T} \underline{\underline{M}} \hat{T} \ddot{u}_{E Q}(t) d t \\
E_{G}=-\int_{\underline{u}(0)}^{\underline{u}(t)} \underline{\underline{M}} \underline{T} g d \underline{u}=-\underline{u}(t)^{T} \underline{\underline{M}} \underline{\underline{T}} g
\end{gathered}
$$

Since the structure is under horizontal earthquake loads and gravity loads, $\underline{T}$ and $\underline{T}$ are transformation column vectors to map the seismic and gravity accelerations onto the horizontal and vertical directional masses and displacements, respectively. If $N$ is the number of total nodes in the system, the response vectors $\underline{u}, \underline{\dot{u}}, \underline{\ddot{u}}$ and the restoring force $f_{s}$ would become $3 N$ by 1 vectors assuming each node has three degree-of-freedoms (i.e., horizontal, vertical and rotational, respectively). Similarly, the system properties $\underline{\underline{M}}$ and $\underline{\underline{C}}$ are $3 N$ by $3 N$ matrices. The transformation matrix $\underline{T}$ would then become a $3 N$ by 2 matrix, where the first and the second columns are the transformation vectors $\underline{T}$ and $\underline{T}$ respectively:

$$
\underline{\underline{T}}=[\underline{\hat{T}} \underline{\underline{T}}]=\left[\begin{array}{llllllllll}
1 & 0 & 0 & 1 & 0 & 0 & 1 & 0 & 0 & \ldots \\
0 & 1 & 0 & 0 & 1 & 0 & 0 & 1 & 0 & \ldots
\end{array}\right]^{T}
$$

During the earthquake excitations, the earthquake energy applied on the structure is dissipated by the work done by the damping and hysteretic forces. The damping and hysteric energies are irrecoverable while elastic and kinetic energies are recoverable energies. If all of the individual energy components are gathered together, the energy balance of a structure in Eq. (3) is now described as:

$$
E_{K}+E_{D}+E_{S}=E_{E Q}+E_{G}
$$

Historically, it has been common to neglect the gravity energy in most dynamic analyses of building structures that do not purport to be predicting collapse. Akiyama [23] stated that gravity energy can be considered as a release of potential energy as result of P-Delta effects, and that it takes part in the total resistance of a structure against a seismic excitation. Therefore, gravity energy can be also shown on the left side of the energy balance as in Eq. (15), however, gravity energy is assumed as a part of the input energy applied to the system in this study.

$$
E_{K}+E_{D}+E_{S}-E_{G}=E_{E Q}
$$

\subsection{Indication of dynamic instability by structural gravity energy}

The accumulation of permanent lateral drifts during a strong ground shaking may induce large P-delta effects that would eventually lead to dominant gravity forces and eventual collapse [24]. 
Therefore, this paper proposes a new collapse criterion which compares the amount of dynamic the vertical static loads during the dynamic analysis. In this work, we have investigated the incidence of gravity energy exceeding dynamic input energy as an indicator of the domination of gravity loads over dynamic loads, thus triggering collapse.

For example, Fig. 4 presents the input-energy-time histories of the validated SDOF model constructed for the test by Kanvinde [8] under the ground motion record of Obregon Park from 1994 Northridge earthquake. At the scale of 0.8 (Fig. 4a), the intensity of the ground motion is not strong enough to trigger the large geometric effects in the frame. Therefore, the structure achieves a steady state in terms of the gravitational energy, which is found insignificant when compared to the quantity of dynamic input energy coming from the ground motion. By contrast, at the scale of 1.0, for which dynamic instability occurs, the geometric nonlinearities in the structure become critical. This causes the frame to show very large displacement in vertical directions and experience a sudden increase in gravitational energy, which eventually exceeds the dynamic input energy.

Fig. 5 shows the structural response of a rotational spring in the frame by Kanvinde [8] (the spring at the bottom of the right column (BR) of the frame in Fig. 1a) in a "phase plot", i.e. rotation versus rotational velocity, for the ground motion input scales of 0.8 (Fig. 5a) and 1.0 (Fig. 5b). Circular marks in the plots indicate negative eigenvalues of tangential system stiffness, i.e., static instability at the corresponding time step in the analysis. For the scale of 0.8 (Fig. 5a), the trajectory at first produces stable paths dissipating around the initial equilibrium state, then exhibits static instability by negative eigenvalues. However, the trajectory converged to another equilibrium state by the end of the analysis. On the contrary, at a scale of 1.0 (Fig. 5b), the trajectory in the phase space loses static stability first, and then fails to achieve another equilibrium state. This confirms that the static instability condition or a trend in phase plot alone cannot indicate the dynamic instability condition. Using the proposed energy-based approach one can overcome this challenge, and does not need to check each degree-of-freedom of the structure. Moreover, the approach may facilitate developing a mathematical description of dynamic instability, which can be particularly useful for stochastic analysis of collapse requiring quantitative and accurate detection during repeated computational simulations.

The developed energy-based criterion is tested using a more sophisticated MDOF example. Fig. 6 shows the input energy components for the validated MDOF model developed for the case study by Lignos et al. [10] under the ground motion record of Canoga Park from the 1994 Northridge Earthquake applied in the experiment. In order to get the energy time histories, the MDOF model of the test frame was continuously loaded for intensity scales of $0.4,1.0,1.5,1.9$, and 2.2, following the test procedure. While the gravity energy is steady in the non-collapse case at the scale of 1.9 (Fig. 6a), it increases significantly and exceeds the dynamic energy in the collapse case at a scale of 2.2, indicating dynamic instability due to loss in resistance against the applied gravity loads. Fig. 7 shows the time histories of several energy components for the same MDOF model under the test ground motion with the collapse-inducing intensity scale of 2.2. As seen in the figure, the potential energy released near structural collapse state far-exceeds the earthquake (dynamic) input energy stored in the structure, and a large amount of potential energy released near collapse state is transformed into kinetic energy. The rest of the released potential energy is reintroduced into the structure through damping energy and strain energy during the deformation of the destabilized components. 
The proposed energy-based collapse criterion was verified by checking energy time histories of the three test case studies [8-10] using totally 78-ground motion records provided by Haselton and Deierlein [20]. In most of the cases, as the seismic intensity increases up to the level of dynamic instability, the gravity energy rapidly increases and far-exceeds the dynamic input energy. In few ground motion cases, actual collapse, i.e., dynamic instability, was observed at the very next step of the intensity scaling following the exceedance of the gravity energy. This occurs rarely, and it is noted that the proposed criterion is mainly indicating significant rapid increase in gravity energy, which is able to indicate the dynamic instability accurately in all collapse simulations.

In a notable energy-based approach by Szyniszewski and Krauthammer [25], the change in the kinetic energy of a system is considered as an indicator of collapse as part of their investigations of energy flow during progressive collapse of steel framed buildings. They stated that the sudden release of the gravitational energy leads to transient motions and kinetic energy, and a building loses its stable configuration if the kinetic energy is not completely dissipated by the structure. Similar to their study, the collapse criterion proposed in the paper also uses an energy concept to identify dynamic instability, but the approach described herein considers gravity energy from the P-delta effects as result of accumulation of permanent large lateral drifts in the system during a strong ground shaking. When the system undergoes large seismic amplitudes, the kinetic energy can show high variations but eventually goes to zero once the structure comes to rest. By contrast, gravity energy can inform us about the status of the system, showing through an energy measure of the permanent impact of P-delta effects on the system at the end of the time history analysis.

\section{Comparison of collapse limit-states}

The collapse capacity of a structure evaluated by existing IDA-based approaches may be sensitive to a particular selection of ground motions as well as possible paths taken by IDA curves. Deterministic criteria have been proposed by Vamvatsikos and Cornell (2002), including an IM-based criterion established as the decrease of the slope of an IDA curve to $20 \%$ of the initial IDA slope (Fig. 8a) and a DM-based criterion established as an exceedance of the drift capacity of $10 \%$ (Fig. 8b). In this section, these IDA-based collapse limit-states are compared to the energy-based collapse criterion (Fig. 8c) to investigate the merits of the proposed approach.

\subsection{Comparison of IDA-based collapse limit-states with new energy collapse criterion}

The validated model developed for the case study by Lignos et. al [10] was utilized here to perform nonlinear dynamic analyses using 78 far-field ground motion set by Haselton and Deierlein [20]. Fig. 8d shows the IDA curves of spectral acceleration $(\mathrm{Sa})$ vs. maximum lateral inter-story drift ratios (IDR) obtained from the validated OpenSees model. Traditional IDAbased criteria are compared to the new "energy criterion" based on the maximum intensity level observed before the dynamic instability occurs, i.e., gravity energy exceeds dynamic energy. For this example, it is clearly seen that the energy criterion consistently predicts a larger drift at collapse. This demonstrates that the meaning of collapse assumed for the traditional criteria may not indicate the actual collapse capacity, but rather may be more representative of collapse prevention.

Moreover, it is noted that the IDA-based criteria could produce more than one collapse capacity estimate for a given ground motion. For example, the IM-based criterion and DM-based 
criterion define two and three collapse points for the cases shown in Fig. 9a and Fig. 9b, respectively. The new energy-based criterion employs an earthquake energy quantity that evolves monotonically as the intensity increases and a gravity energy quantity that typically shows significant increase only at the intensity level of collapse, thus providing only one point of structural collapse. This can be also shown from Eq. (11) and Eq. (12). While the gravity energy depends on the instant change of vertical displacement, the earthquake energy is an integral quantity aggregating the impact of the seismic actions on the structural response. Also, the time history analyses performed for the case studies of Kanvinde [8], Rodgers and Mahin [9], and Lignos et al. [10] under all 78-ground motions confirmed this finding [13].

In Fig. 8d, significant variability is observed in the estimated collapse capacities for all criteria due to the effect of randomness in the selected ground motions on collapse behavior. For comparison purpose, histograms of collapse capacities in terms of IDR and $S a$ obtained from both IM-based and DM-based criteria normalized to those from the energy criterion are given in Fig. 10 and Fig. 11, respectively. In both figures, larger standard deviation (std) is observed in the estimated drift capacity than the estimated intensity level. This is the case especially when the IM-based criterion is used (Fig. 10b), because the deformation capacity from the DM-based criterion (Fig. 11b) depends on a predetermined threshold value, giving the same drift capacity for each collapse intensity level. Since the DM-based criterion estimates intensity levels (square markers in Fig. 8d) that are close to those by the energy criterion (circular markers in Fig. 8d), less dispersion is observed in the DM-based intensity levels (Fig. 11a) as compared to the IMbased intensity levels (Fig. 10a).

Fig. 12 and Fig. 13 show correlation between the collapse capacities in terms of IDR and $S_{a}$ resulting from the IM-based criterion and the proposed energy criterion, and those resulting from the DM-based criterion and the proposed energy criterion, respectively. As expected (from Fig. 8d), a strong correlation is observed in the estimated intensity measures for both the DM-based and IM-based criteria (Fig. 12a and Fig. 13a). The sample correlation coefficients are 0.97 (IMbased vs energy-based), and 0.95 (DM-based vs energy-based). Strong correlation means that the collapse capacities estimated in terms of spectral acceleration tends to be linearly proportional to those based on actual dynamic instability. However, it should be noted that it does not necessarily mean that they are close to each other in general. Due to much sensitivity of story drift capacities to ground motion details, a much lower correlation value is observed for the collapse capacities given in terms of the drift ratio when IM-based approach and energy-based approach are compared (Fig. 12b). Since the DM-based criterion assumes a fixed threshold value on the drift ratio, correlation analysis is meaningless in Fig. 13b. In summary, the collapse-level intensity measure, e.g., $S_{a}$ predicted by existing IDA-based approaches correlates well with the dynamic instability level predicted by the energy-based approach, while the collapse-level damage measure, e.g., IDR, is not well predicted by the existing approaches. These figures highlight the potential strengths of using energy-based collapse criterion. The evaluation of damage within a structural system under seismic forces benefits from a quantitative descriptor that captures the physical condition of the complete system, such as is expressed as a function of global and cumulative structural responses in the energy quantities [13]. These figures thus highlight the potential benefits of developing new quantities that may be used with the new energy-based criterion to better represent the collapse performance. 
460 Next, sensitivity of IDA results obtained by the IM-based or DM-based criteria with respect to the threshold values is investigated by normalizing the results by those from the energy criterion. Several assumed threshold values are considered on initial IDA slope percentage and drift capacity for the IM-based or DM-based criteria respectively. For example, Fig. 14 presents the sensitivity of IDA results using the IM-based criterion with various values from $10 \%$ to $30 \%$ of the initial IDA slope. In Fig. 14a, the $S a$ level by the IM-based criterion gets close to the $S a$ capacity determined by the energy criterion as the slope of the IM-based criterion decreases. The standard deviation in the ratios of $S a$ levels (dashed lines) also decreases as the slope percentage value decreases. Using the same slope percentage values for the IM-based criterion, sensitivity analyses performed on the damage measure IDR (Fig. 14b) show larger standard deviations in the ratios of IDR levels (dashed lines), as expected. However, the standard deviation does not show as much difference as in the previous case. This may be due to the characteristics of the IDA curves in which the slope of the curves may show a sudden increase or decrease towards collapse.

Similarly, sensitivity analyses are performed for the DM-based criterion with different IDR threshold values from 5 to $15 \%$ as shown in Fig. 15. Note that the number of data points considered in the sensitivity analyses decreases as the threshold value for drift capacity increases more than $10 \%$. For example, although totally 78 ground motions are at first considered in the analyses, the frame reaches to collapse earlier than the presumed drift threshold $15 \%$ for 13 ground motions. The results of sensitivity analyses show that DM-based criterion gives more sensitive and biased estimates with more rapid changes observed in mean ratios of IDA results (dotted lines in Fig. 15 comparing to the ones in Fig. 14). While deviations in the ratios of $S a$ levels increase as the threshold value decreases (Fig. 15a), deviations in the ratios of IDR levels tend to decrease (Fig. 15b). This is because the results determined based on DM-based criterion give intensity capacities far from the points obtained based on the energy criterion (squared data points in Fig. 8d) but give the same drift capacity for each collapse intensity level. Therefore, IDA results by DM-based criterion with a smaller threshold value such as $5 \%$ significantly underestimates the collapse capacity according to the energy criterion.

In summary, the sensitivity analysis results show that collapse capacities identified by the DM-based and IM-based criteria are sensitive to the subjective values assumed for IDA curve slope and damage thresholds as compared to the energy-based criterion.

\section{Conclusions}

A new energy-based collapse criterion is proposed to predict collapse of structures caused by dynamic instability due to failure of the structural system under gravity loads. This criterion is proposed as an alternative to existing criteria based on subjective threshold values to predict collapse using incremental dynamic analysis curves. Using computational simulations validated by three collapse tests, input energy components released into the structure due to earthquake ground motions and applied gravity loads were compared to develop the new collapse criterion which indicates the dynamic instability accurately and effectively. Based on the observations on energy-component analyses, the new collapse criterion has been defined as a boundless increase in gravity energy. A quantitative indication of structural collapse has been then defined as the gravity energy exceeding dynamic input energy, that is, $\boldsymbol{E}_{\boldsymbol{G}} \geq \boldsymbol{E}_{\boldsymbol{E} \boldsymbol{Q}}$. It has also been found that while IDA-based traditional criteria can define more than one collapse capacity within an IDA

504 curve, the new energy criterion typically identifies a unique structural collapse capacity. In 
addition, these energy terms are aggregated quantities of responses of structural components that can represent global seismic performance of the structural system. This criterion was validated using 2D-computational models that in turn were compared to the results of three sets of shaketable tests of reduced-scale steel structures. The new criterion can serve as a reliable indicator for the purpose of collapse prediction, thus forming the basis for identification of key parameters that would help describe structural collapse, and development of fragility relations and other approaches for assessing the risk of collapse for ductile multi-story moment frame structures showing planar motion under unidirectional horizontal seismic loads with comparable hysteretic behavior to these collapse experiments. Future research is warranted to test and validate the criterion for a variety of $2 \mathrm{D}$ and $3 \mathrm{D}$ full scale-structural frames under both horizontal and vertical ground motions $[26,27]$.

\section{Acknowledgements}

This material is based upon work supported by the National Science Foundation under Grant No. CMMI-1000666, the University of Illinois at Urbana-Champaign, and Northeastern University. The second author also acknowledges the support from the Integrated Research Institute of Construction and Environmental Engineering at Seoul National University, and the National Research Foundation of Korea (NRF) Grant (No. 2015R1A5A7037372), funded by the Korean Government (MSIP).

\section{References}

[1] Vamvatsikos, D., and Cornell, C.A. (2002). "Incremental Dynamic Analysis," Earthquake Engineering and Structural Dynamics, 31(3), 491-514.

[2] FEMA P-695 (2009). Quantification of Seismic Performance Factors, FEMA P-695 Report, prepared by the Applied Technology Council for the Federal Emergency Management Agency, Washington, DC.

[3] Mehanny S.S., and Deierlein G.G. (2000). Modeling and Assessment of Seismic Performance of Composite Frames with Reinforced Concrete Columns and Steel Beams, Report No. 136, The John A. Blume Earthquake Engineering Center, Stanford University, Stanford, CA.

[4] Park, Y.-J., and Ang, A.H.-S. (1985). "Mechanistic Seismic Damage Model for Reinforced Concrete,' Journal of Structural Engineering, ASCE, 111(4), 722-739.

[5] Kratzig, W.B., Meyer, I.F., and Meskouris, K. (1989). "Damage Evolution in Reinforced Concrete Members under Cyclic Loading," Proceedings of $5^{\text {th }}$ International Conference on Structural Safety And Reliability, ASCE, New York, USA. Vol (2). 795-802.

[6] Massumi A, and Monavari B. (2013). "Energy based Procedure to Obtain Target Displacement of Reinforced Concrete Structures," Structural Engineering and Mechanics, 48(5), 681-695.

[7] Open System for Earthquake Engineering Simulation (2004). Pacific Earthquake Engineering Research Center, University of California, Berkeley, CA.

[8] Kanvinde, M (2003). "Methods to Evaluate the Dynamic Stability of Structures-Shake Table Tests and Nonlinear Dynamic Analyses," EERI Annual Student Paper Competition, Earthquake Engineering Research Institute, Oakland, Calif.

[9] Rodgers, J.E., and Mahin, S.A. (2004). Effects of Connection Hysteretic Degradation on the Seismic Behavior of Steel Moment-Resisting Frame, Pacfic Earthquake Engineering 
Research Center Report, PEER 2003/13.

[10] Lignos, D.G., Krawinkler, H., and Whittaker, A.S. (2008). "Shaking Table Collapse Tests of Two Scale Models of a 4-Story Moment Resisting Steel Frame," Proceedings of the 14th World Conference on Earthquake Engineering, Beijing, China, October 12-17.

[11] Lee. K. and Foutch, D.A. (2004). "Performance Evaluation of Damaged Steel Frame Buildings Subjected to Seismic Loads," Journal of Structural Engineering, 130(4), 588599.

[12] Lee. K., and Foutch, D.A. (2002). "Seismic Performance Evaluation of Pre-Northridge Steel Frame Buildings with Brittle Connections," Journal of Structural Engineering, 128(4), 546555.

[13] Deniz, D. (2015). Stochastic Prediction of Collapse of Building Structures under Seismic Excitations, Ph.D. Dissertation, University of Illinois at Urbana-Champaign, Urbana, Illinois.

[14] Deniz, D., Song, J., and Hajjar, J. F. (2015). "Energy-based Seismic Collapse Risk Assessment of Structures," Proceedings of the 12th International Conference on Applications of Statistics and Probability in Civil Engineering (ICASP12), Vancouver, Canada.

[15] Menegotto, M., and Pinto, E. (1973). "Method of Analysis for Cyclically Loaded Reinforced Concrete Plane Frames Including Changes in Geometry and Non-Elastic Behavior of Elements under Combined Normal Force and Bending," Proceedings of IABSE Symposium, Lisbon, Portugal.

[16] Denavit M.D., and Hajjar J.F. (2013). Description of geometric nonlinearity for beamcolumn analysis in OpenSees, Report No. NEU-CEE-2013-02, Department of Civil and Environmental Engineering, Northeastern University, Boston, MA.

[17] Lignos, D.G., Krawinkler, H., Whittaker, A., (2009). "Contributions to Collapse Prediction of Steel Moment Frames Through Recent Earthquake Simulator Collapse Tests", Proceedings 3rd International Conference on Advances in Experimental Structural Engineering, October 15-16, San Francisco, CA.

[18] Lignos, D.G. and Krawinkler, H. (2012). Sidesway Collapse of Deteriorating Structural Systems under Seismic Excitations, Report No. 177, The John A. Blume Earthquake Engineering Center, Department of Civil and Environmental Engineering, Stanford University, Stanford, CA.

[19] Ibarra, L.F., Medina, R.A., and Krawinkler, H. (2005). "Hysteretic Models that Incorporate Strength and Stiffness Deterioration", International Journal for Earthquake Engineering and Structural Dynamics, 34:11, 1489-1511.

[20] Haselton, C.B., and Deierlein, G.G. (2007). Assessing Seismic Collapse Safety of Modern Reinforced Concrete Frame Buildings, Report No. 156, The John A. Blume Earthquake Engineering Center, Stanford University, Stanford, CA.

[21] Bernal, D. (1998). "Instability of Buildings during Seismic Response," Engineering Structures, 20(4-6), 496-502.

[22] Uang, C. M. and Bertero, V. V. (1990). "Evaluation of Seismic Energy in Structures," Earthquake Engineering and Structural Dynamics, 19,77-90.

[23] Akiyama, H. (2002). "Collapse Modes of Structures under Strong Motions of Earthquake," Annals of Geophysics, 45(6), 791-798.

[24] Jennings, P. C., and Husid, R. (1968). "Collapse of Yielding Structures during Earthquakes," Journal of Engineering Mechanics Division, 94(5), 1045-1065. 
605

606

607

608

609

610

611

612

613

614

615

616

617

618

619

620

621

622

623

624

625

626

627

628

629

630

631

[25] Szyniszewski, S. and Krauthammer, T. (2012). "Energy Flow in Progressive Collapse of Steel Framed Buildings," Engineering Structures, 42, 142-153.

[26] Yamada, S., Suita, K., Tada, M., Kasai, K., Matsuoka, Y., and Shimada, Y. (2008). "Collapse Experiment on a 4-Story Steel Moment Frame: Part 1 Outline of Test Results," The $14^{\text {th }}$ World Conference on Earthquake Engineering, Beijing, China, October 12-17.

[27] Suita, K., Yamada, S., Tada, M., Kasai, K., Matsuoka, Y., and Shimada, Y. (2008). "Collapse Experiment on a 4-Story Steel Moment Frame: Part 2 Detail of Collapse Behavior," The $14^{\text {th }}$ World Conference on Earthquake Engineering, Beijing, China, October 12-17. 


\section{List of Figures}

633 Fig. 1. a) Test frame structure by Kanvinde [8]; b) structural model configuration; c) spring model details and d) displacement time history results from OpenSees for Obregon Park ground motion [1 in $\approx 2.54 \mathrm{~cm}]$.

Fig. 2. a) Shake-table test by Lignos et al. $[10,17]$; b) structural model developed for the test frame with circles indicating the location of rotational springs at the element ends; c) the component model assumed for rotational springs.

Fig. 3. Comparison of experimental test results by Lignos et al. $[10,18]$ and OpenSees simulations of lateral displacement time history at the top of the frame for Canoga Park ground motion [1 in $\approx 2.54 \mathrm{~cm}]$.

Fig. 4. Input energy components of Kanvinde's experiment [8] near collapse under the test earthquake of the 1994 Northridge earthquake at Obregon Park a) non-collapse case at the ground motion scale of 0.8 and b) collapse case at the ground motion scale of 1.0.

Fig. 5. Phase plots of structural response of the bottom-right (BR) rotational spring in the computational simulation model developed for Kanvinde's experiment [8] at the ground motion-scale of a) 0.8 and b) 1.0 .

650

651

652

653

654

655

656

657

658

659

660

661

662

663

664

665

666

667

668

669

670

671

672

673

674

675

676

677

678

Fig. 6. Input energy components for the test case by Lignos et al. [10] near collapse under the test earthquake of the 1994 Northridge earthquake at Canoga Park a) non-collapse case at the ground motion scale of 1.9 and b) collapse case at the ground motion scale of 2.2 .

Fig. 7. Time histories of energy components for the MDOF model of the case study of Lignos et al. [10] near collapse under the test earthquake of Canoga Park at the scale of 2.2.

Fig. 8. a) IM-based, b) DM-based, c) energy-based criteria, and d) IDA curves obtained for the test case study of Lignos et al. [10] using the 78 far-field ground motion set [20].

Fig. 9. Multiple collapse capacity estimates when using a) IM-based and energy criteria, and b) DMbased and energy criteria.

Fig. 10. Histograms for collapse capacities in a) intensity measure $S a$ and b) damage measure IDR obtained by IM-based criterion normalized to collapse capacities by the energy criterion.

Fig. 11. Histograms for collapse capacities in a) intensity measure $S a$ and b) damage measure IDR obtained by DM-based criterion normalized to collapse capacities by the energy criterion.

Fig. 12. Correlation between IM-based criterion and the energy criterion based on the levels of measures: (a) $S a$ and (b) IDR.

Fig. 13. Correlation between DM-based criterion and the energy criterion based on the levels of measures: (a) $S a$ and (b) IDR.

Fig. 14. Sensitivity analyses on the levels of measures a) $S a$ and b) IDR based on IM-based criterion with different slopes.

Fig. 15. Sensitivity analyses on the levels of measures a) $S a$ and b) IDR based on DM-based criterion with different drift capacity threshold values. 
(a)

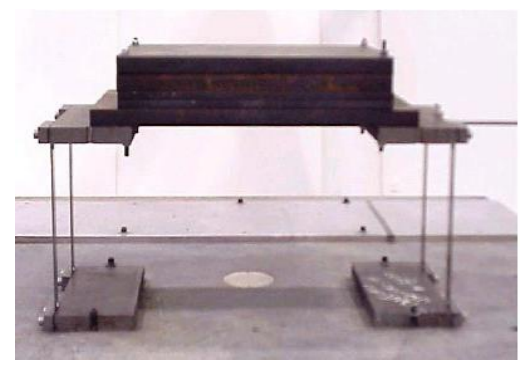

(c)

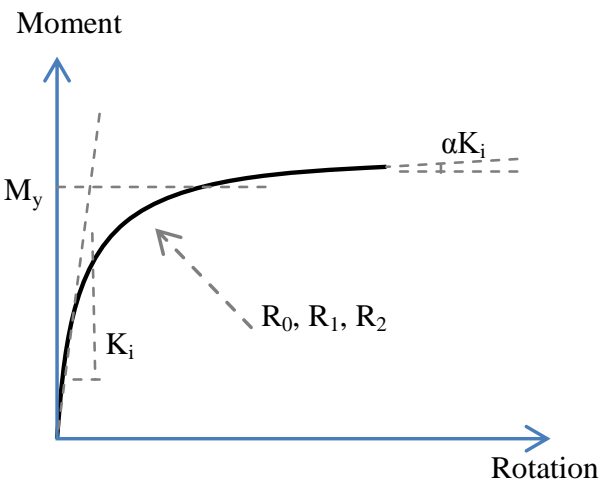

(b)

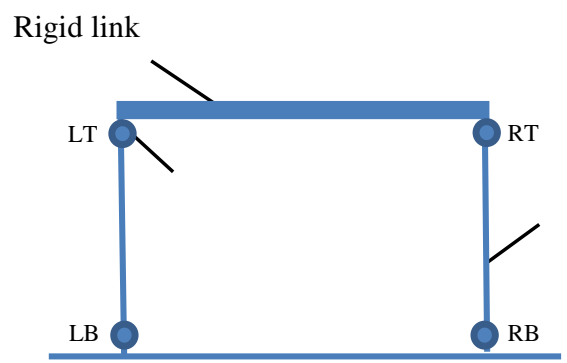

(d)

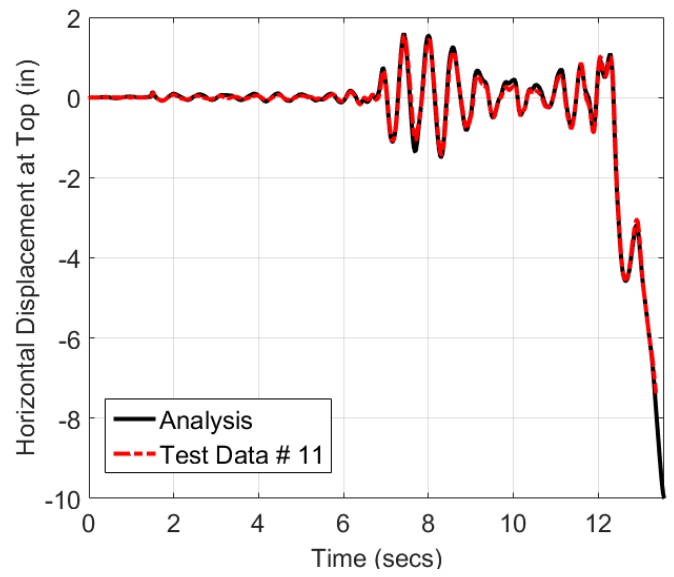

680

681

682

683
Fig. 1. 
(a)

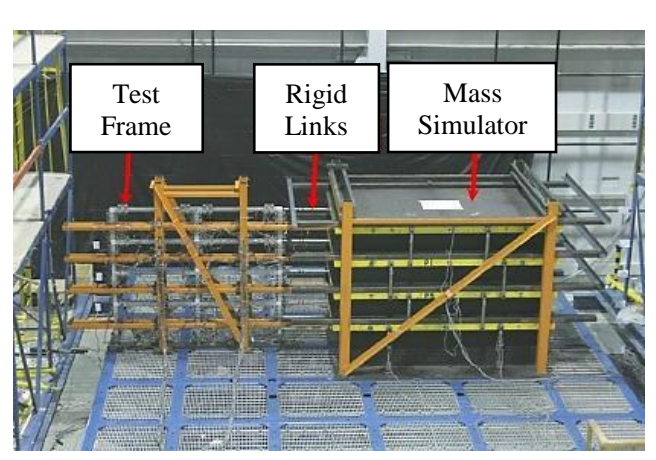

(b)

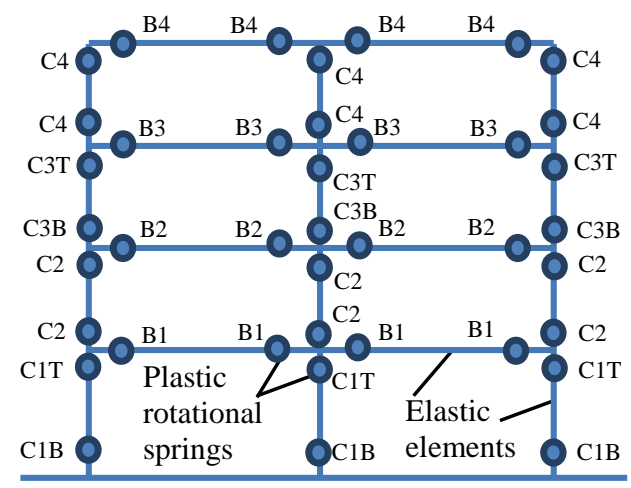

(c)

684

685

686

Fig. 2.

687

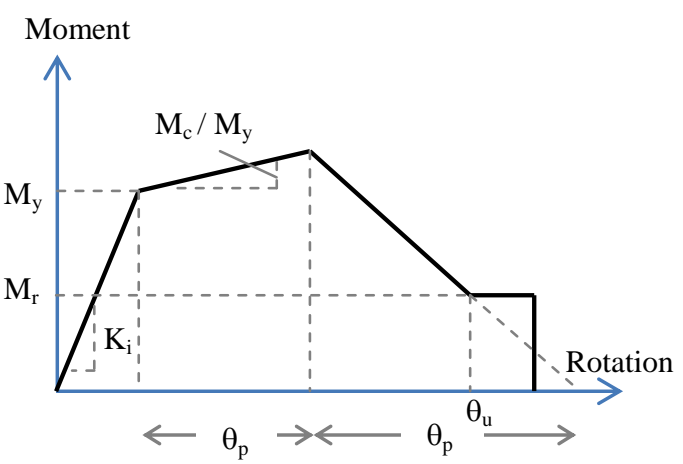



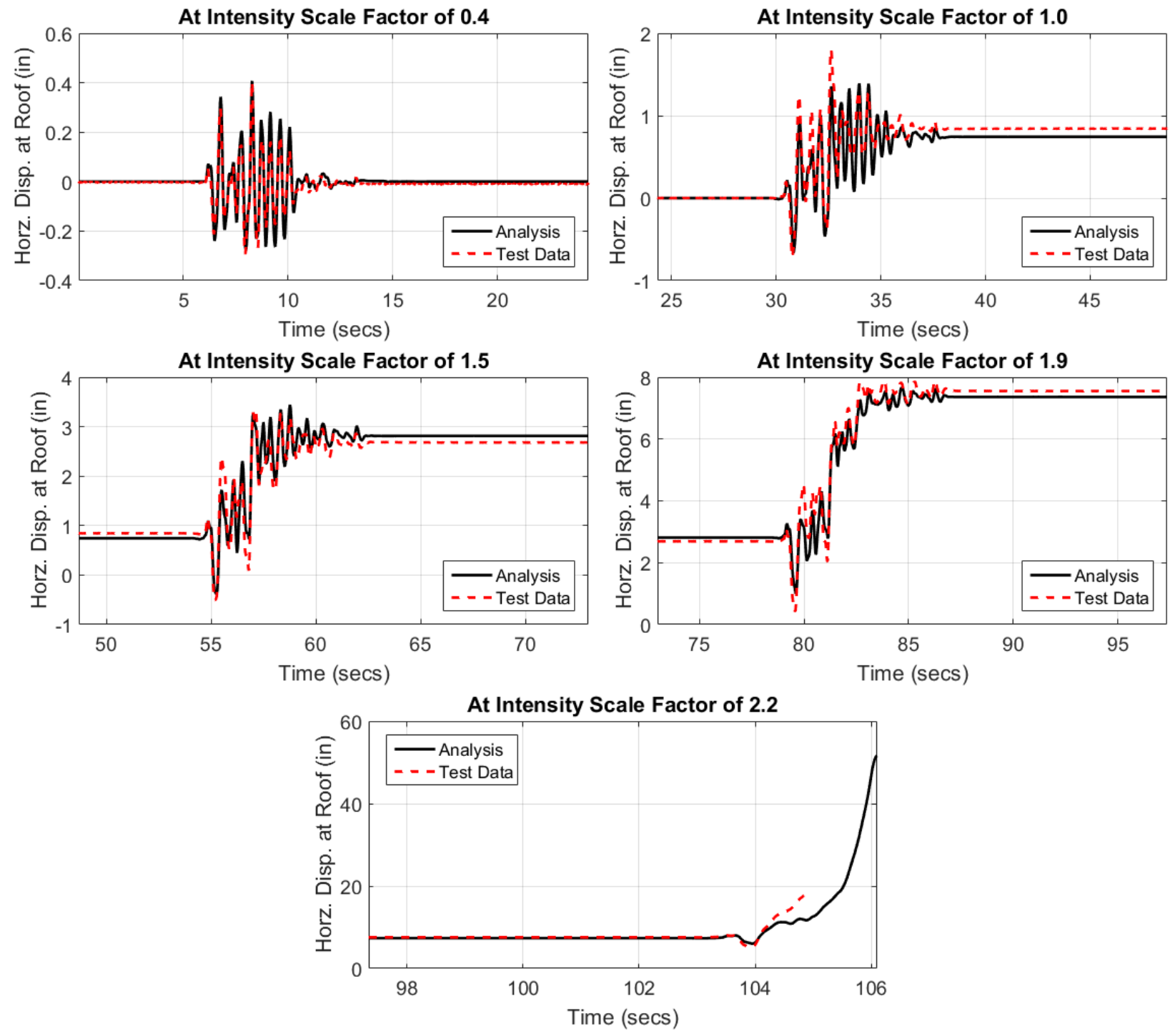

692

693

694

695

696

Fig. 3. 
(a)

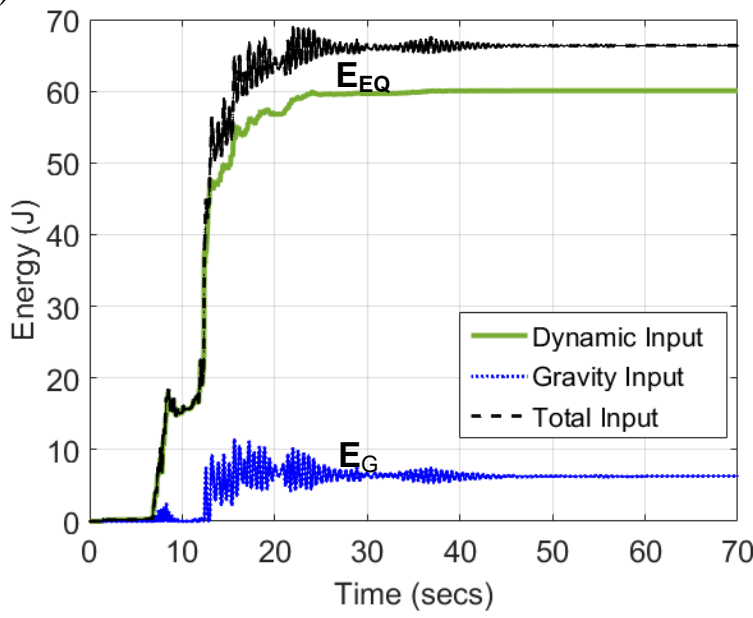

(b)

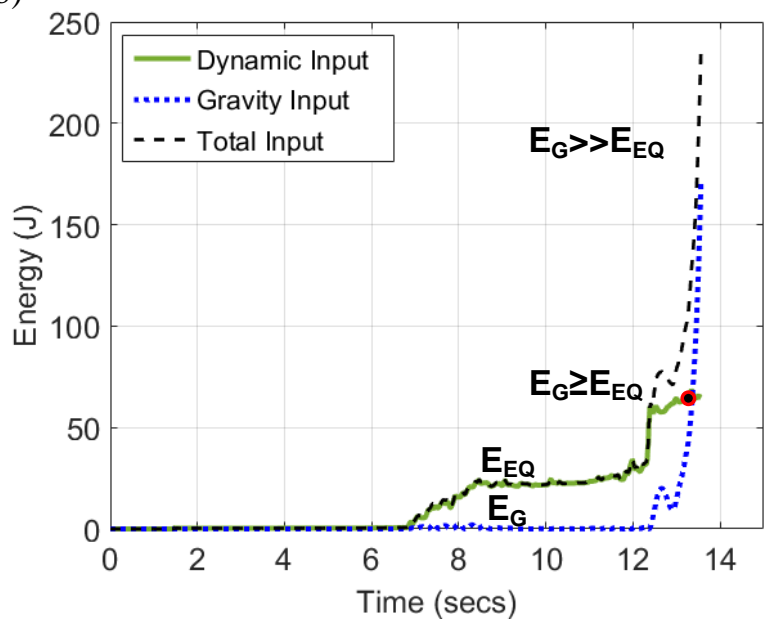

698

699

700

701

702

703

704

705

706

707

708

709

710

711

712

713

714

715

716

717

718

719

720

721

722

723

724

725

726

727

728

729

730

731

732

Fig. 4. 

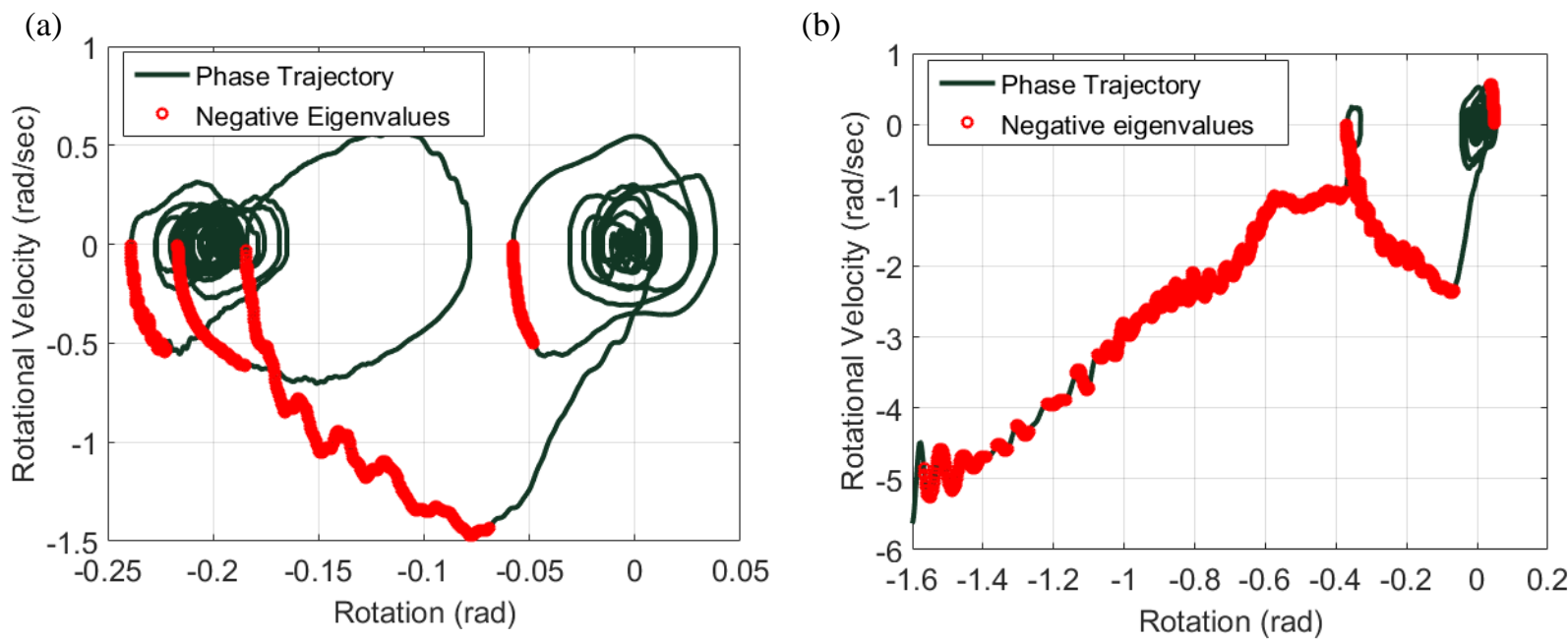

733

734

735

736

737

738

739

740

741

742

743

744

745

746

747

748

749

750

751

752

753

754

755

\section{Fig. 5.}


760

761

762

763

764

765

766

767

768

769

770

771

772

773
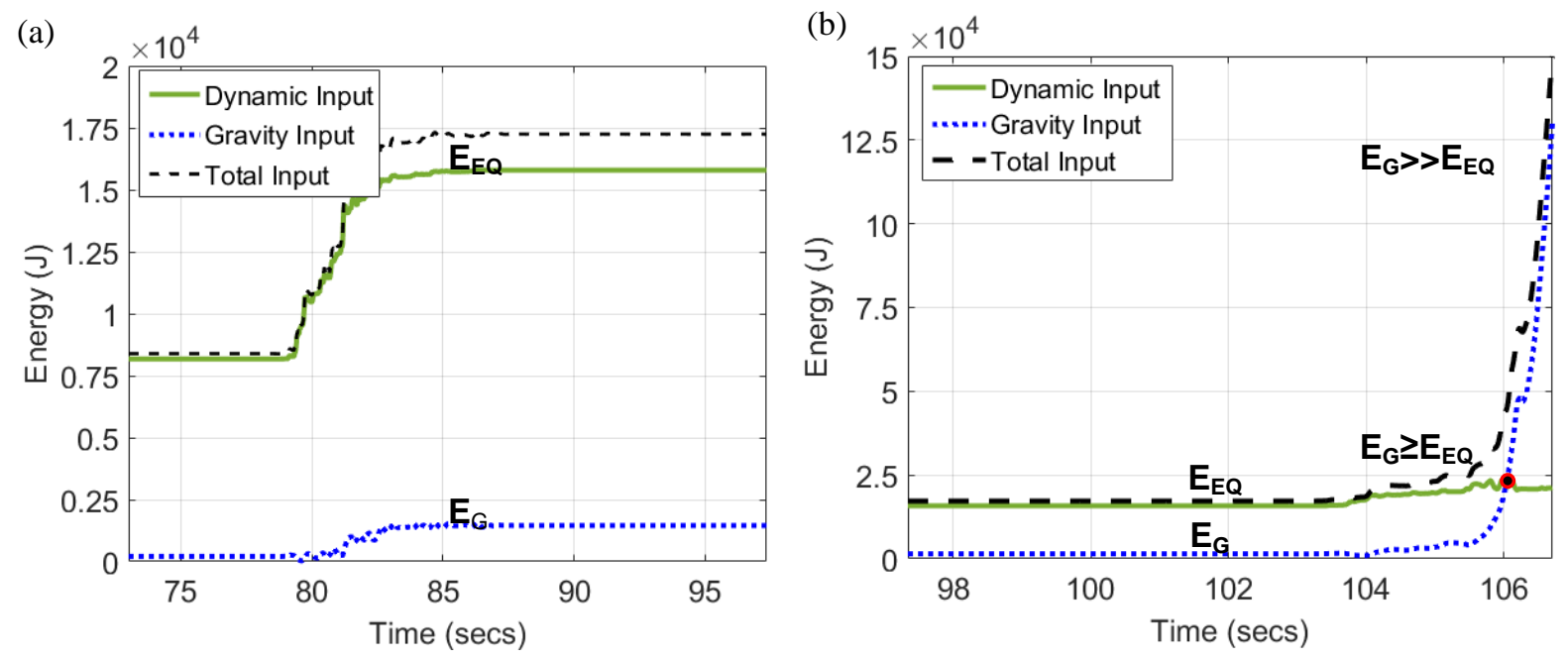

Fig. 6. 


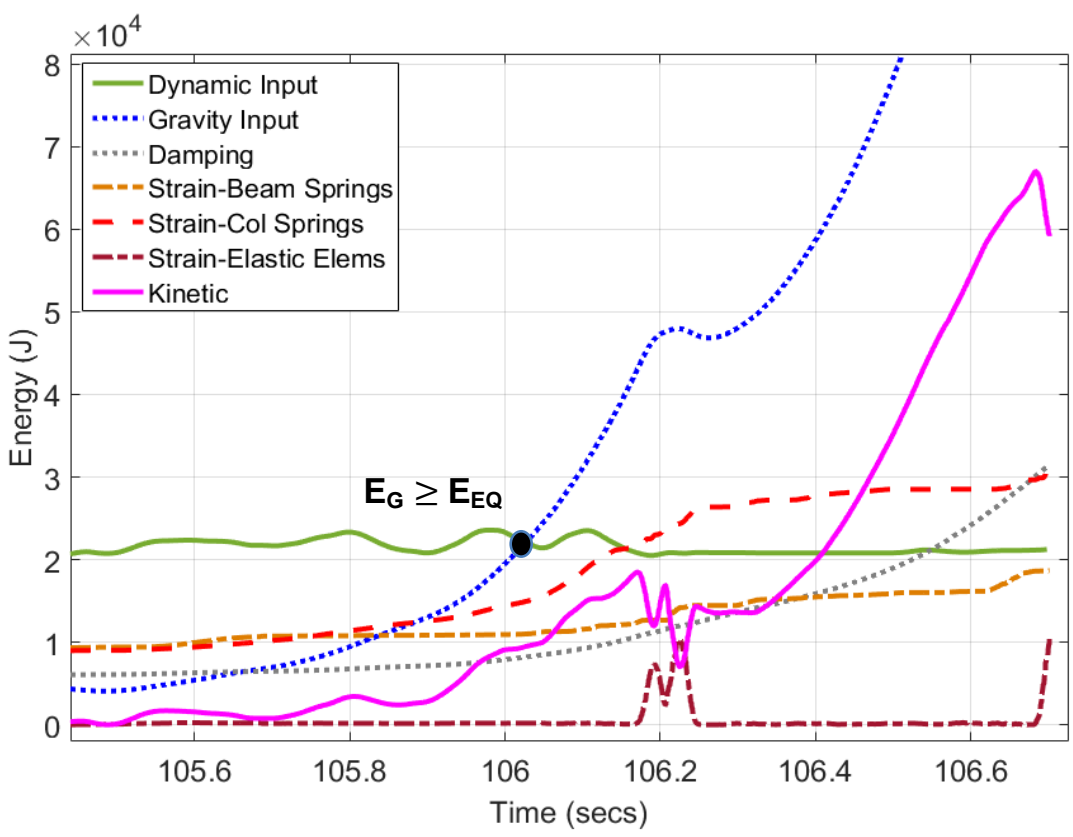

778

Fig. 7.

880

82 
(a)

(b)

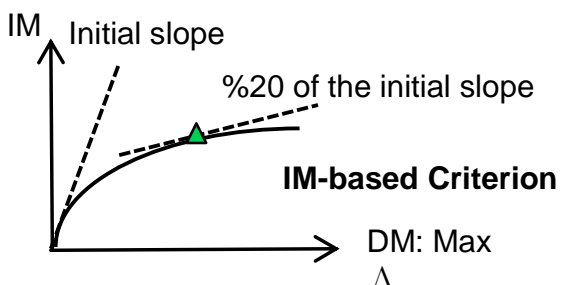

(c)

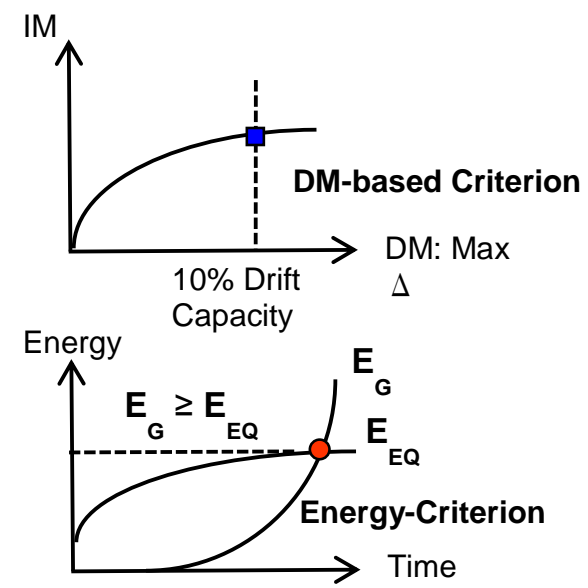

(d)

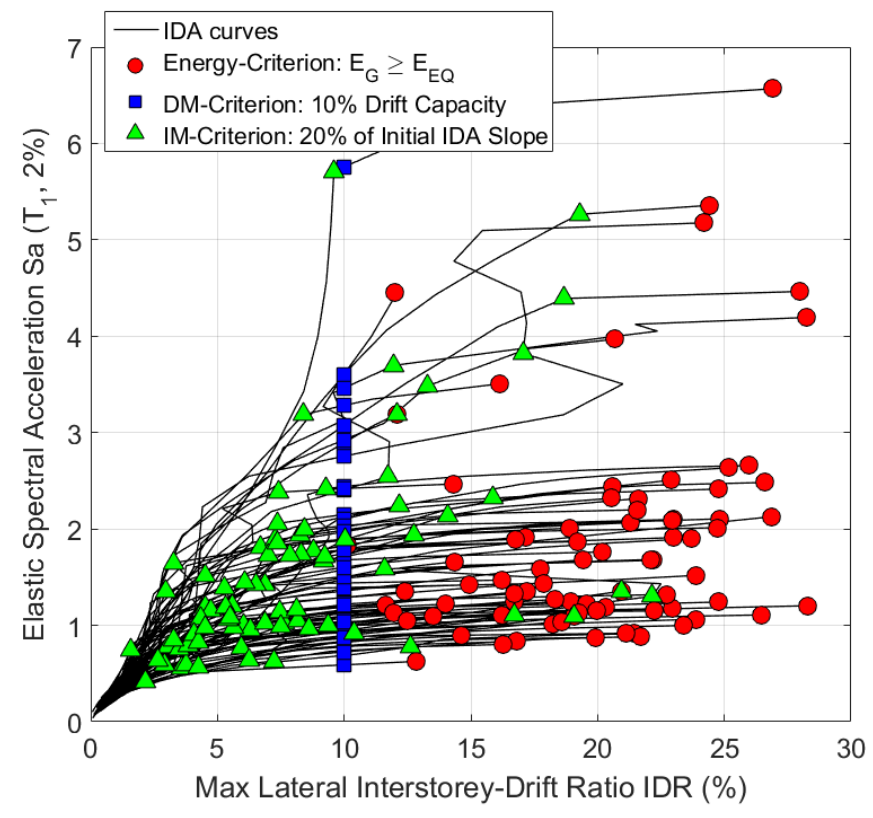

784

785

786

787

788

789

790

791

792

793

794

795

796

Fig. 8. 
(a)

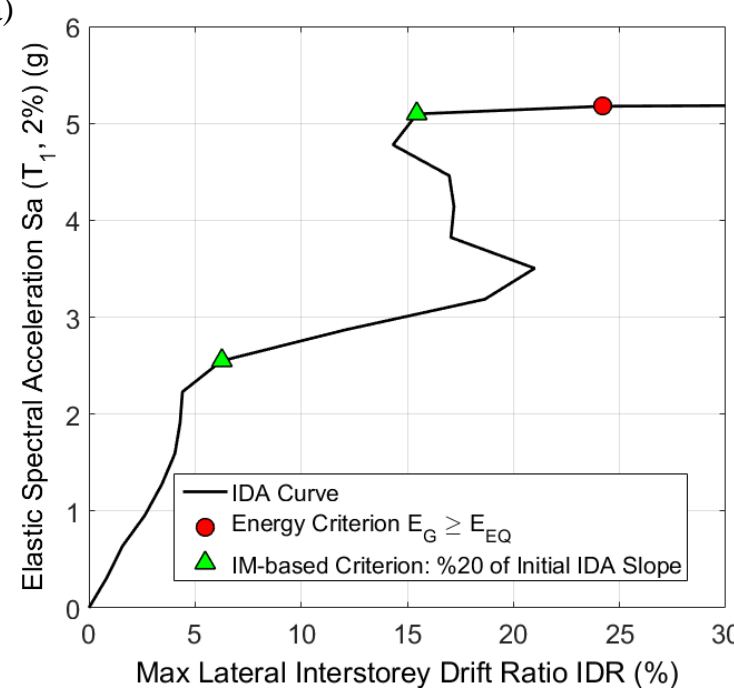

797

798

799

800

801

802

803

804

805

806

807

808

809

810

811

812

813

814

815

816

817

818

Fig. 9. (b)

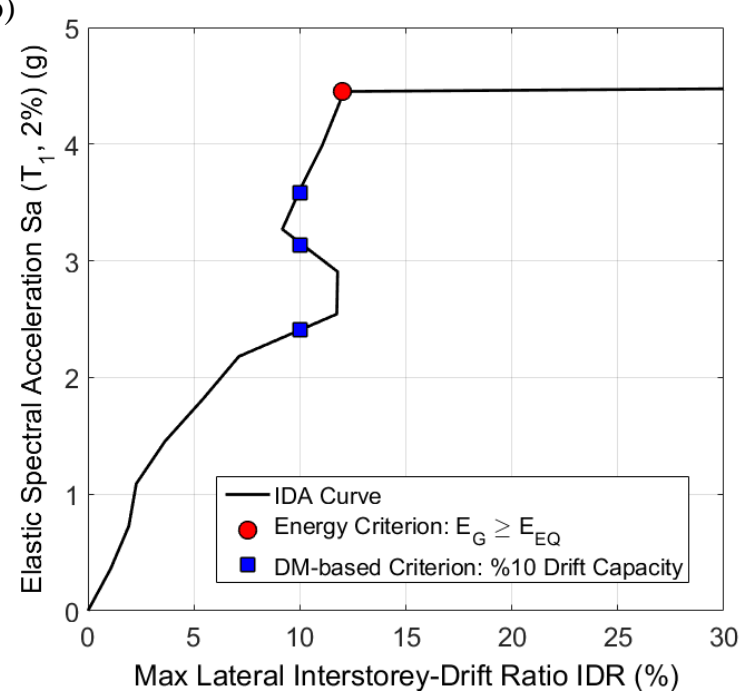


(a)

819

820

821

822

823

824

825

826

827

828

829

830

831

832

833

834

835

836

837

838

839

840

841

842

843

844

Fig. 10. (b)

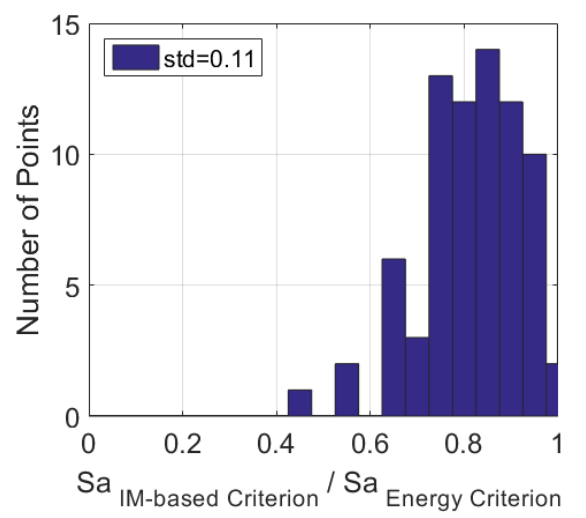

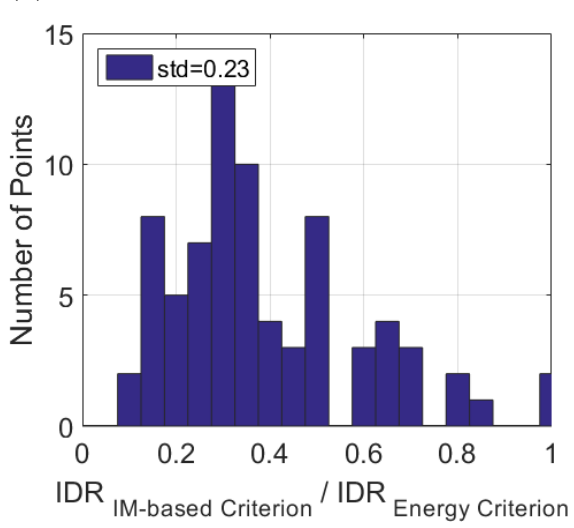


(a)

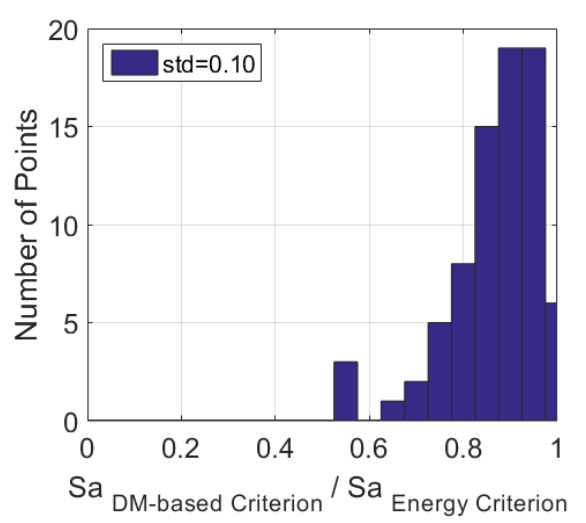

(b)

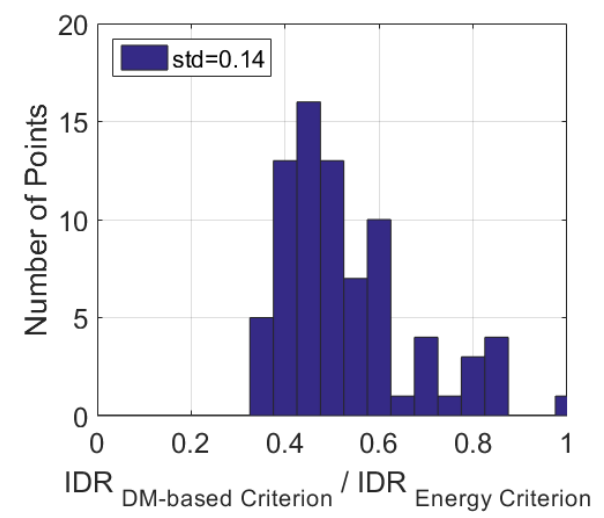

846

847

848

849

850

851

852

853

854

855

856

857

858

859

860

861

862

863

864

865

866

867

868

Fig. 11.

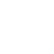


(a)

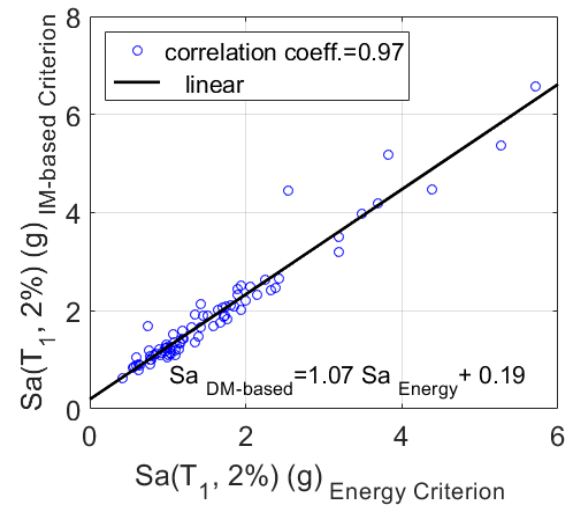

(b)

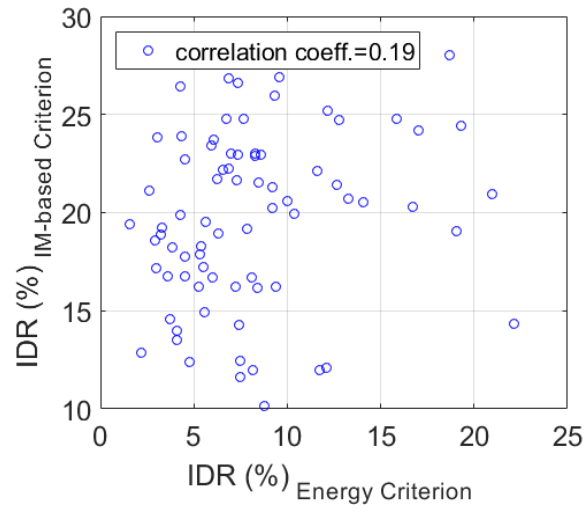

869

870

871

872

873

874

875

876

877

878

879

880

881

882

883

884

885

886

887

888

889

890

891

892

893

894

Fig. 12.

\section{Fig. 12.}


(a)

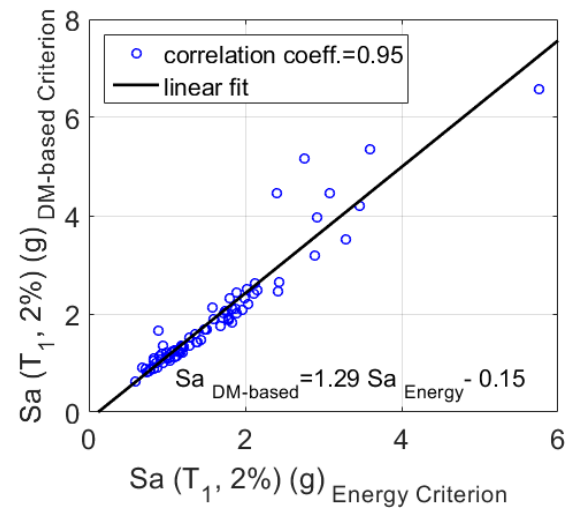

(b)

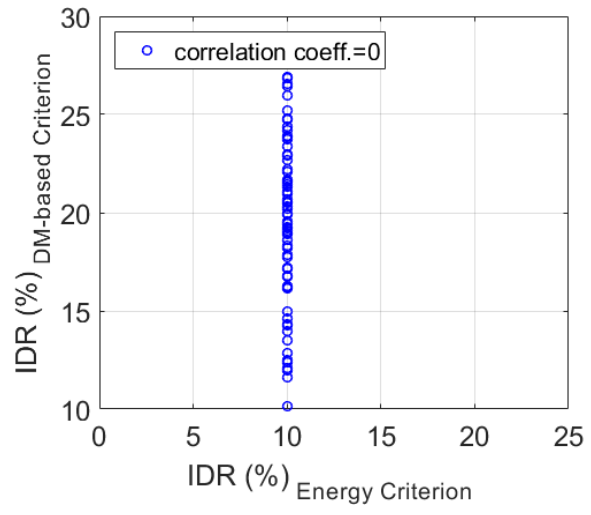

895
896

897

898

899

900

901

902

903

904

905

906

907

908

909

910

911

912

913

914

915

916

Fig. 13. 
(a)

917

918

919

920

921

922

923

924

925

926

927

928

929

930

931

932

933

934

935

936

937

938

Fig. 14. (b)
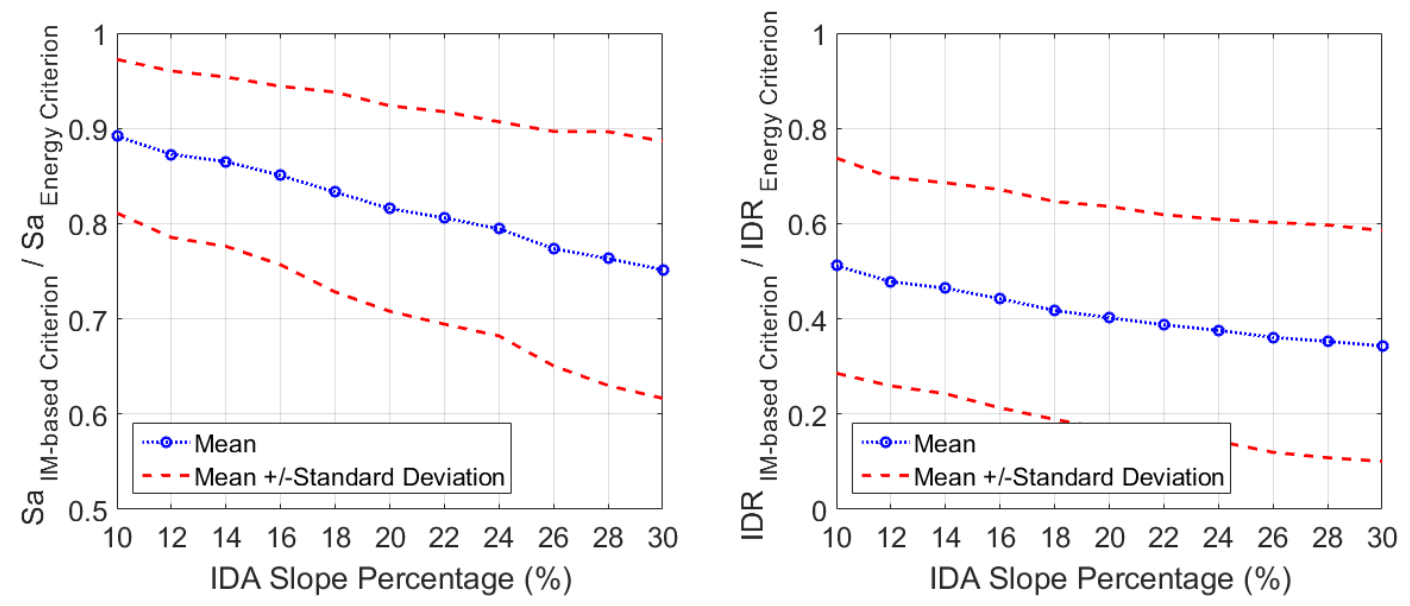
(a)

939

940

941

942

943

944

945

946

Fig. 15. (b)
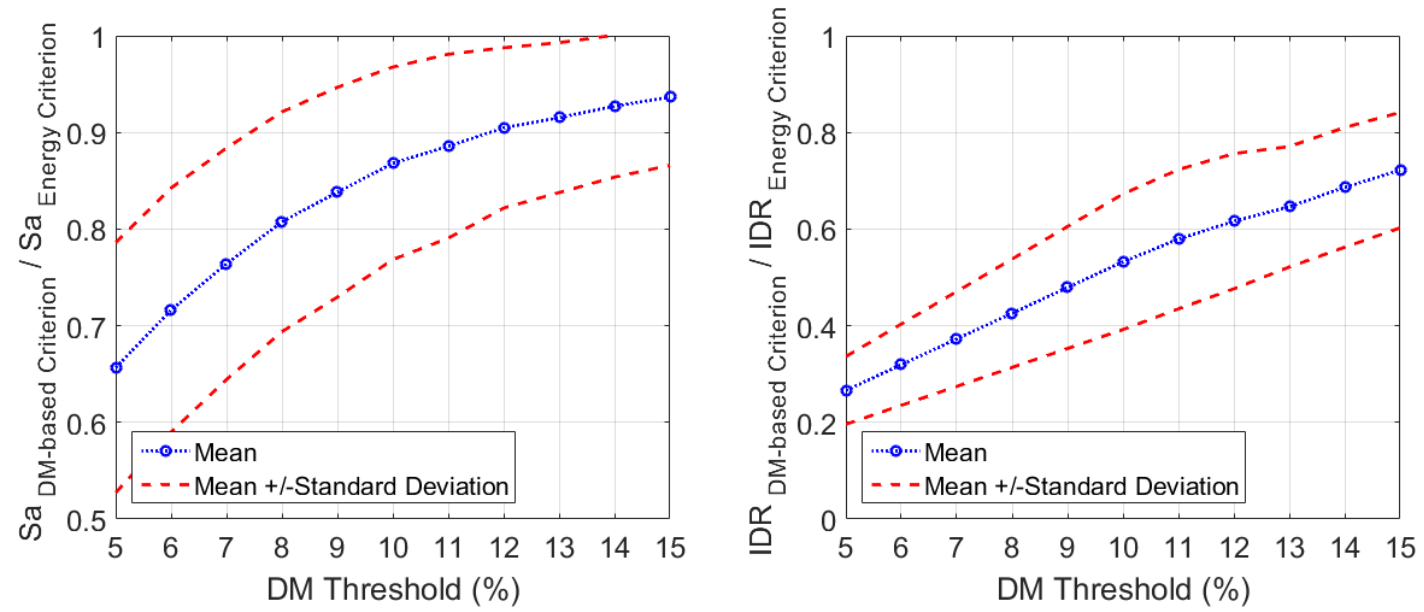